\title{
Knowledge Discovery for Transonic Regional-Jet Wing through Multidisciplinary Design Exploration*
}

\author{
Kazuhisa CHIBA**, Shigeru OBAYASHI $^{* * *}$ and Hiroyuki MORINO ${ }^{\dagger}$ \\ ** Japan Aerospace Exploration Agency \\ 7-44-1 Jindaiji-Higashi, Chofu, Tokyo 182-8522, Japan \\ E-mail: chiba@chofu.jaxa.jp \\ *** Tohoku University \\ 2-1-1 Katahira, Sendai 980-8577, Japan \\ $\dagger$ Mitsubishi Heavy Industries, Ltd. \\ 10 Oye-cho, Minato-ku, Nagoya 455-8515, Japan
}

\begin{abstract}
Data mining is an important facet of solving multi-objective optimization problem. Because it is one of the effective manner to discover the design knowledge in the multiobjective optimization problem which obtains large data. In the present study, data mining has been performed for a large-scale and real-world multidisciplinary design optimization (MDO) to provide knowledge regarding the design space. The MDO among aerodynamics, structures, and aeroelasticity of the regional-jet wing was carried out using high-fidelity evaluation models on the adaptive range multi-objective genetic algorithm. As a result, nine non-dominated solutions were generated and used for tradeoff analysis among three objectives. All solutions evaluated during the evolution were analyzed for the tradeoffs and influence of design variables using a selforganizing map to extract key features of the design space. Although the MDO results showed the inverted gull-wings as non-dominated solutions, one of the key features found by data mining was the non-gull wing geometry. When this knowledge was applied to one optimum solution, the resulting design was found to have better performance compared with the original geometry designed in the conventional manner.
\end{abstract}

Key words : Knowledge Discovery, Multidisciplinary Design Exploration, Regional Jet Aircraft, Data Mining

\section{Introduction}

Although design optimization, especially practical multidisciplinary design optimization (MDO), has become an important manner for engineering, the most significant point is the extraction of the knowledge in design space. The result obtained by multi-objective (MO) optimization result using evolutionary algorithm is not a sole solution but a set of optimum solutions. That is, as MO optimization result is only figure enumeration, there is insufficient information for designers. However, the set of optimum solutions can be considered hypothetical design database. Recently, data mining technique is applied for the optimization result as the hypothetical design database to obtain the fruitful design knowledge efficiently [1,2]. As the combination between the optimization and data mining is a sequence process, it is called as MO design exploration (MODE) [3] or multidisciplinary design exploration (MDE) instead of MDO in the present study. Although Ref. [3] shows the introduction of MODE and the outline of its application of the regional jet aircraft, this paper reveals the concrete knowledge of its application, the decision process of the final configuration is described. Moreover, the reason why the optimization did not obtain the result which data mining extracted, the importance of MDE is also described.

"Received 19 Apr., 2007 (№. 07-0193) [DOI: 10.1299/jamdsm.2.396] 
The objective of this study is to apply the MDE system to a large-scale and practical aircraft design problem, and to acquire the beneficial knowledge in the design space to employ data mining which is an emerging area of computational intelligence. In the present study, a self-organizing map (SOM) [4,5] as a data mining technique was employed. SOM suggested by Kohonen is one of neural network models, and it can serve as a cluster analyzing tool for high-dimensional data. The cluster analysis of the objective function values will help to identify design tradeoffs and influence of design variables. In this study, the data mining was applied to a large-scale and real-world MDE problem for a regional jet aircraft [6] in the field of aeronautics and space engineering, and then knowledge in the multidisciplinary design space is acquired.

\section{MDO Problem}

\subsection{Objective Functions}

In this system, minimization of the block fuel at a required target range derived from aerodynamics and structures was selected as a primary objective function. In addition, two more objective functions were considered - minimization of the maximum takeoff weight and minimization of the difference in the drag coefficient between two Mach numbers, which are cruise Mach and target maximum operating Mach number (MMO), to prevent decrease MMO.

\subsection{Geometry Definition}

First, the planform was given by Mitsubishi Heavy Industries, Ltd. The front and rear spar positions were fixed in the structural shape based on the initial aerodynamic geometry. The wing structural model was substituted with shell elements.

The design variables were related to airfoil, twist, and wing dihedral. The airfoil was defined at three spanwise cross-sections using the modified PARSEC [7] with nine design variables $\left(x_{u p}, z_{u p}, z_{x x_{u p}}, x_{l o}, z_{l o}, z_{x x_{l o}}, \alpha_{T E}, \beta_{T E}\right.$, and $r_{L E_{l o}} / r_{L E_{u p}}$ ) for each cross-section as shown in Fig. 1. The twists were defined at six spanwise locations, and then wing dihedral positions were defined at kink and tip locations. The twist center was set on the trailing edge in the present study. The entire wing shape was thus defined using 35 design variables. The detail of design variables is summarized in Table 1. In the present study, the geometry of each individual was generated by the unstructured dynamic mesh method [8] using displacement from the initial geometry.

\subsection{Constraints}

The five constraints were considered in the optimizer. The first three were geometrical constraints, while the last two were constraints for flight condition as follows: (1) the distribution of the parameter $\Delta y$ to describe leading-edge geometry was constrained in the spanwise direction to prevent abrupt stall characteristics. Here, $\Delta y$ denotes an airfoil upper surface or-

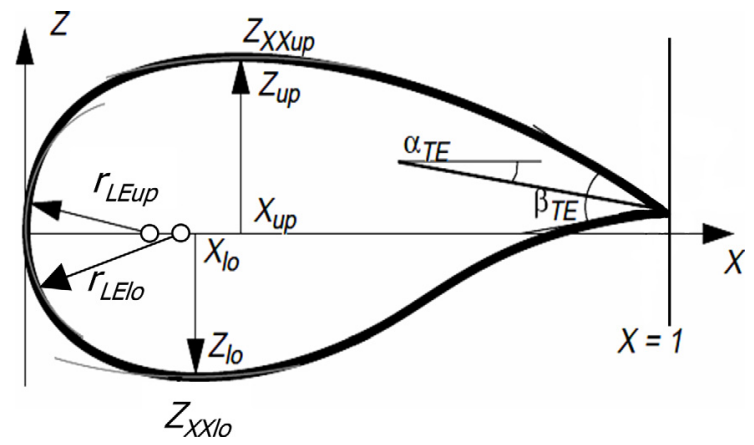

Fig. 1 Illustration of the modified PARSEC airfoil shape defined by nine design variables. 
Table 1 Detail of design variables.

\begin{tabular}{ccl}
\hline serial number & & correspondent design variable \\
\hline 1 to 9 & PARSEC airfoil & $\begin{array}{l}35.0 \% \text { semispan location } \\
\left(x_{u p}, z_{u p}, z_{x x_{u p}}, x_{l o}, z_{l o}, z_{x x_{l}}, \alpha_{T E}, \beta_{T E}, r_{L E_{l o}} / r_{L E_{u p}}\right)\end{array}$ \\
10 to 18 & PARSEC airfoil & $55.5 \%$ semispan location \\
19 to 27 & PARSEC airfoil & $77.5 \%$ semispan location \\
\hline 28 to 33 & Twist angle & $19.3 \%, 27.2 \%, 35.0 \%, 55.5 \%, 77.5 \%, 96.0 \%$ \\
\hline 34,35 & Dihedral & $35.0 \%, 96.0 \%$ \\
\hline
\end{tabular}

dinate at $6 \%$ chord from the leading edge minus the ordinate at $0.15 \%$ chord, (2) rear spar heights were greater than required for housing of the control surfaces, (3) the lower and upper surfaces of the spars changed monotonically in the spanwise direction, (4) the lift coefficients increased monotonically with increasing Mach number to satisfy target lift divergence Mach number (MLD), and (5) the evaluated fuel for the given range was less than the wing fuel volume.

\subsection{Optimizer}

Adaptive range multi-objective genetic algorithm (ARMOGA) [9] is an efficient MO evolutionary algorithm (MOEA) designed for aerodynamic optimization and multidisciplinary design optimization problems using high-fidelity computational fluid dynamics (CFD) solvers with large computational time. ARMOGA has range adaptation based on population statistics, and thus the population is re-initialized every $N$ generations so that the search region adapts toward more promising regions. Because of the re-initialization, ARMOGA can be used with a small population size similar to Micro-GA [10]. ARMOGA can be employed to obtain the non-dominated solutions efficiently because of the concentrated search of the probable design space, while keeping diversity.

In the present ARMOGA, the fitness value of each solution is determined by Fleming and Fonseca's Pareto-ranking method coupled with the fitness sharing approach [11]. Each individual is assigned a rank according to the number of individuals dominating it. The assigned fitness values are divided by the niche count, which is calculated by summing the sharing function values. To find the Pareto solutions more effectively, the so-called best $-N$ selection [12] is also implemented. After determination of shared fitness values for all individuals, the Stochastic Universal Selection (SUS) [13] is applied to select better solutions for producing a new generation. Blended crossover (BLX- $\alpha)$ [14] and polynomial mutation methods [15] are adopted for crossover and mutation.

\subsection{Evaluation Method}

The optimizer generates eight individuals per generation, and evaluates aerodynamic and structural properties of each design candidate as follows: (1) structural optimization is performed to jig shape to realize minimum wing weight with constraints of strength and flutter requirements using NASTRAN. And then, weights of wing box and carried fuel are calculated, (2) static aeroelastic analysis is performed at three flight conditions to determine the aeroelastic deformed shapes (1G shape) using the Euler solver and NASTRAN, (3) aerodynamic evaluations are performed for the 1G shapes using the Navier-Stokes solver, and (4) flight envelope analysis is performed using the properties obtained as above to evaluate the objective functions. Using the objective functions, the optimizer generates new individuals for the next generation via genetic operations, such as selection, crossover, and mutation.

In the present study, MSC. NASTRAN ${ }^{\mathrm{TM}}$ [16] which is a high-fidelity commercial software is employed for the structural and aeroelastic evaluations. Besides, the in-house unstructured mesh solver named as TAS-Code $[17,18]$ is used to evaluate aerodynamic performance such as $C_{L}, C_{D}, L / D$, and $C_{M p}$ using Euler and N-S equations. It is notable that $C_{L}$ denotes the lift coefficient of the fuselage-wing configuration, $C_{D}$ denotes the drag coefficient, $L / D$ is the lift-drag ratio, and $C_{M p}$ denotes the pitching moment coefficient around the center of 
gravity of the fuselage-wing configuration.

\section{Optimization Results}

The population size was set to eight, and then roughly 70 Euler and 90 Reynolds-averaged Navier-Stokes (N-S) computations were performed in one generation for computational fluid dynamics (CFD) evaluation. It took roughly one hour of CPU time for single Euler computation, and it also took roughly nine hours for single N-S computation on NEC SX-5 and SX-7 vector machines per processing element. The population was re-initialized every five generations for the range adaptation. First, evolutionary computation was performed for 17 generations. Then, the evolutionary operation was restarted using eight non-dominated solutions extracted from all solution of 17 generations, and two more generations were computed. A total evolutionary computation of 19 generations was carried out. The total of all solutions was 130 individuals and nine non-dominated solutions were generated. The evolution may not converge yet. However, the results were satisfactory because several non-dominated solutions achieved significant improvements over the initial design. Furthermore, a sufficient number of solutions were searched such that the sensitivity of the design space around the initial design could be analyzed. This will provide useful information for designers.

The evaluated solutions show that the non-dominated front was generated between the block fuel and the drag divergence, there was a tradeoff between the block fuel and the drag divergence. As the non-dominated solutions did not comprise Pareto front between other combinations, there were no global tradeoff between these combinations of the objective functions. Although the wing box weight tends to increase as compared with that of the initial geometry, the block fuel can be reduced. Thus, the aerodynamic performance can redeem the penalty due to the structural weight. An individual on the non-dominated front was selected, indicated as 'optimized', and then the optimized geometry was compared with the initial geometry. The shock wave of the optimized geometry was weaker than that of the initial geometry in the vicinity of the $35.0 \%$ spanwise location indicating the wave drag reduction. Moreover, the vorticity of the wing wake of the optimized geometry near the $35.0 \%$ spanwise location was weaker than that of the initial geometry as shown by helicity contours. Therefore, these figures show that the shape change near the $35.0 \%$ spanwise location, i.e., the shape modification in the vicinity of the kink is effective to reduce the drag.

\section{Data Mining}

Data mining and knowledge discovery is the new field to extract the knowledge from database including the data which statistical analysis cannot treat. It has the sense to transform analysis results into the concrete proposal. In this study, SOM was used. It is an unsupervised learning, nonlinear projection algorithm from high to low-dimensional space. This projection is based on self-organization of a low-dimensional array of neurons. In the projection algorithm, the weights between the input vector and the array of neurons are adjusted to represent features of the high dimensional data on the low-dimensional map. The close two patterns are in the original space, the closer is the response of two neighboring neurons in the low-dimensional space. Thus, SOM reduces the dimension of input data while preserving their features. In this study, SOMs were generated by using commercial software Viscovery ${ }^{\circledR}$ SOMine 4.0 Plus [19] produced by Eudaptics GmbH. Although SOMine is based on the general SOM concept and algorithm, it employs an advanced variant of unsupervised neural networks, i.e., Kohonen's Batch SOM [20,21]. The algorithm consists of two steps that are iterated until no more significant changes occur: search of the best-matching unit $c_{i}$ for all input data $\left\{\boldsymbol{x}_{i}\right\}$ and adjustment of weight vector $\left\{\boldsymbol{m}_{j}\right\}$ near the best-matching unit. The trained SOM is systematically converted into visual information [22,23].

\section{Data-Mining Results}

\subsection{Tradeoff Analysis of the Design Space}

All of the solutions have been projected onto the two-dimensional map of SOM. Figure 2 


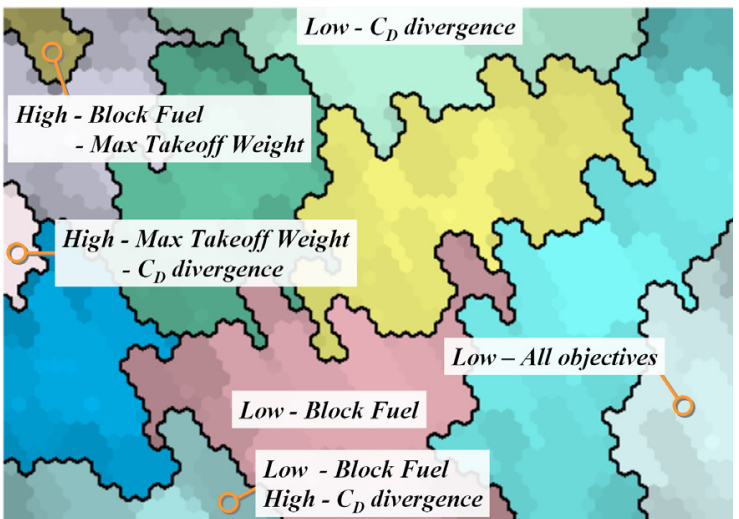

Fig. 2 SOM of all solutions in the three-dimensional objective function space with 11 clusters.

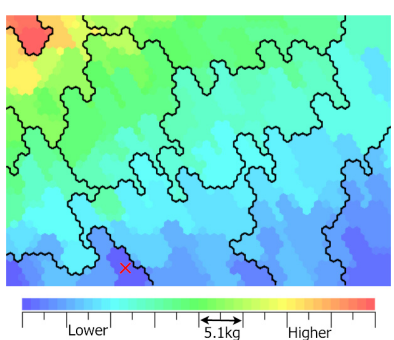

(a) The block fuel

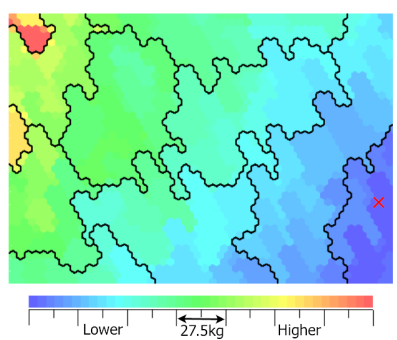

(b) The max takeoff weight

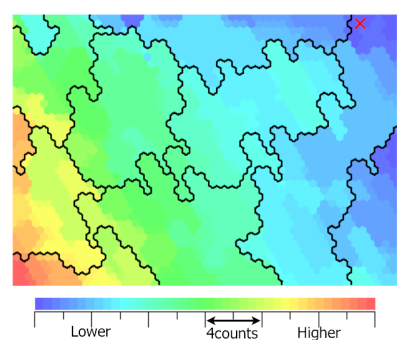

(c) The $C_{D}$ divergence

Fig. 3 SOM colored by the objective functions. The symbol $\times$ denotes the respective extreme non-dominated solutions.

shows the resulting SOM with 11 clusters considering the three objectives. Furthermore, Fig. 3 shows the SOMs colored by the three objectives. These color figures show that the SOM indicated in Fig. 2 can be grouped as follows: The upper left corner corresponds to the designs with high block fuel and maximum takeoff weight. The left center area corresponds to designs with high maximum takeoff weight and $C_{D}$ divergence. The lower left corner corresponds to designs with low block fuel and high $C_{D}$ divergence. Figure 3(a) and Fig. 3(c) show that there is a tradeoff between these two objective functions. The lower center area corresponds to designs with low block fuel. The right hand side corresponds to designs with low $C_{D}$ divergence. As the coloring in Fig. 3(a) is similar to that in Fig. 3(b), there was not a severe tradeoff between the block fuel and the maximum takeoff weight. The lower right corner corresponds to designs with low value of all objectives. Extreme non-dominated solutions are indicated in Fig. 3(a) to (c). As they are in different clusters, the simultaneous optimization of the three objectives is impossible. However, the lower right cluster has relatively low values for all three objectives. Thus, this region of the design space may provide a sweet spot for the present design problem.

\subsection{Effects of Aerodynamic Performance on Objective Functions}

Figure 4 shows the SOMs colored by the aerodynamic performance under transonic cruising flight condition. Figures 4(a) and (b) show the SOMs colored by $C_{L}$ and $C_{D}$, respectively. As these figures show similar coloring, the $L / D$ increase is not so easy. Lower $C_{D}$ values are located in the lower right corner in Fig. 4(b). As this area clusters designs with low value of all objectives, this observation suggests that when all objectives are optimized simultaneously, the $C_{D}$ under the cruising flight condition is also reduced. Furthermore, as the clusters of lower values of the maximum takeoff weight shown in Fig. 3(b) appears on the right hand side of the map, $C_{D}$ can be decreased simultaneously with the maximum takeoff weight. As the area with higher $C_{D}$ shown in Fig. 4(b) generally coincide with the area with 


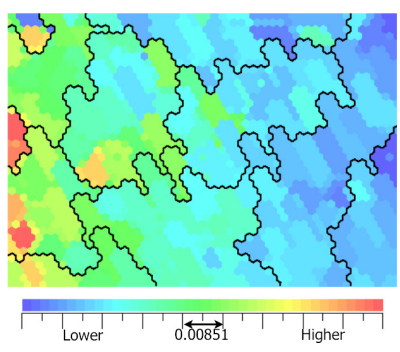

(a) $C_{L}$

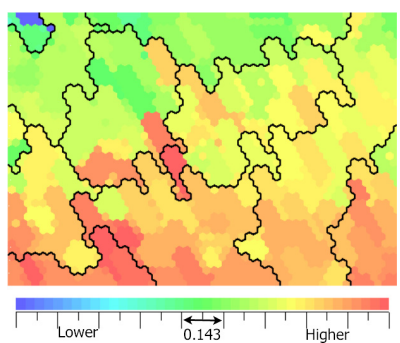

(c) $L / D$

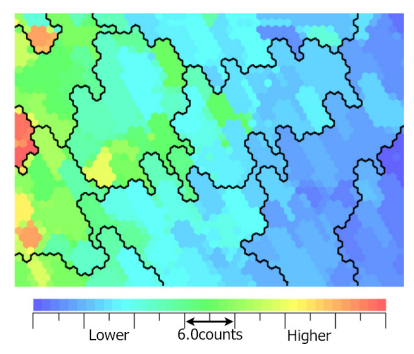

(b) $C_{D}$

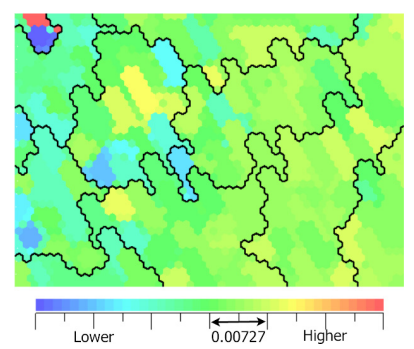

(d) $C_{M p}$

Fig. 4 SOM colored by aerodynamic performance under transonic cruising flight condition.

higher objective function values, $C_{D}$ is a very important performance index.

Figure 4(c) shows the SOM colored by $L / D$; lower values are located in the upper left corner. As the higher values of the block fuel shown in Fig. 3(a) are present at the same location, lower $L / D$ makes the block fuel worse. Furthermore, higher $L / D$ values are located in the lower area shown in Fig. 4(c). As the lower values of the block fuel shown in Fig. 3(a) are present at the same area, higher $L / D$ was effective to decrease the block fuel. However, higher transonic $L / D$ values were not necessarily effective to reduce the block fuel in Fig. 4(c) because not only the cruise condition but also the complete flight profile from takeoff to landing were considered in the present study.

Figure 4(d) shows the SOM colored by $C_{M p}$. When $C_{M p}$ increases and $C_{L}$ decreases and $L / D$ is reduced. $C_{L}$ and $C_{D}$ increase with decreasing $C_{M p}$. That is, a decrease in $C_{M p}$ makes the objective function values worse.

As the resulting SOMs, colored by $C_{L}$ and $C_{D}$ under subsonic flight condition, appear similar to transonic $C_{L}$ and $C_{D}$ shown in Fig. 4(a) and (b), their influences to the objective functions were also the same. That is, the effects of subsonic aerodynamic performance on objective functions might be predicted from the effects of transonic aerodynamic performance in the present study. This fact reveals that the wing is evolved into the shape on which the shock wave is weakened, and then the aerodynamic performance at the transonic condition is resembling that at the subsonic one.

\subsection{Additional Characteristics}

Figure 5 shows the SOM colored by three other characteristic values. Figure 5(a) shows the SOM colored by the constraints of the evaluated fuel mass. The colored value was defined as Value $=$ Volume $_{\text {required fuel }}-$ Volume $_{\text {fuel capacity }}$, where, Volume $_{\text {required fuel }}$ denotes

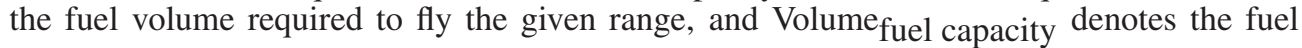
capacity volume that can actually be carried in the wing. When this value is greater than zero, the aircraft cannot fly the given range. As the area with values of over zero corresponds to the area with high maximum takeoff weight, the aerodynamic characteristics and design values that have effects on maximum takeoff weight dominate this constraint.

Figure 5(b) shows the SOM colored by the ranking in the optimizer based on Pareto ranking. As the upper left region has a poorer ranking, larger block fuel and maximum takeoff 
weight as objective functions 1 and 2 dominate the poor ranking. In contrast, the lower left area with higher $C_{D}$ divergence does not have poor ranking. These observations indicate that improvement in $C_{D}$ divergence is not dominated by the specific aerodynamic performance and design variables, and further improvement cannot be achieved by the present problem easily.

Figure 5(c) shows the SOM colored by the angle between inboard and outboard on the upper wing surface for the gull-wing at the kink location. Angles greater and less than $180 \mathrm{deg}$ correspond to gull and inverted gull-wing, respectively. The locations of higher values of this angle as shown in Fig. 5(c) correspond to positions of higher $C_{D}$ under the transonic cruising flight condition shown in Fig. 4(b). However, at angles less than $180 \mathrm{deg}$, there was little correlation between Fig. 4(b) and Fig. 5(c). The inverted gull-wing did not affect aerodynamic performance. The inverted gull-wing is known to have a structural weight increase, which is also observed in the present results. Indeed, the locations of higher angles in Fig. 5(c) had higher maximum takeoff weights as shown in Fig. 3(b). Therefore, non-gull wings should be designed in future.

\subsection{Effects of Design Variables}

Finally, Fig. 6 and Fig. 7 show the SOMs colored by the selected design variables with regard to the PARSEC airfoil parameters at $35.0 \%$ and $55.5 \%$ spanwise locations, respectively. Moreover, Fig. 8 shows the SOM colored by the design variable, twist angle. The design variables can be summarized as follows, taking into consideration the effects on each objective function and aerodynamic performance.

There are no design variables that show large effects on objective function 1 as block fuel. The large twist angles at the $35.0 \%$ spanwise location makes objective function 2 as maximum takeoff weight worse. In addition, large twist angles at the $55.5 \%$ spanwise location increase objective function 3 as $C_{D}$ divergence. However, no design variable of the PARSEC airfoil had apparent effects on any objective functions by itself. As shown later, PARSEC design variables have direct effects on aerodynamic performances. However, the present objective functions are not pure aerodynamic characteristics. Therefore, effects of the design variables on the objective functions were not trivial. There were no design variables and no aerodynamic characteristics that were effective on the sweet spot with relatively low values for all three objective functions. Therefore, the individual that resides in the sweet spot cannot be generated by hand. A correlation between objective function and design variable is desirable when the sensitivity of the design variable is to be investigated; this is one of the important aspects in optimization problems in general.

Next, the effects of design variables on aerodynamic performance were investigated. From the correspondence between Figs. 4, 6, 7, and 8, the effects of respective design variables are summarized in Tables 2 to 4 . These tables indicate that the design variables of the PARSEC airfoil have effects on aerodynamic performance directly. It is noted that the effects of design variables to $C_{D}$ can be predicted from the above results because Figs. 4(a) and (b) are similar. Furthermore, the effects of design variable on aerodynamic performance under the subsonic flight condition can be predicted because the SOMs appeared similar at the transonic and subsonic flight conditions. The leading-edge curvature of PARSEC airfoil at $35.0 \%$ spanwise location was effective to $L / D$ and $C_{M p}$.

The geometry near the $55.5 \%$ spanwise location was not changed markedly with regard to twist angle, as shown in Fig. 8(b). The geometry near the $96.0 \%$ spanwise location was changed to upward twisting. Conversely, the geometry near the $35.0 \%$ spanwise location was changed to downward twisting. The improvement in the vicinity of the $35.0 \%$ spanwise location restrained the shock wave, reducing the wave drag. When the drag decreases, the lift may decrease simultaneously. The lift was increased to compensate for the reduction in the vicinity of the kink so that the angle of attack of the outboard wing was increased although the wing is still twisted down. It should be noted that the angle of attack near the kink had an effect on the transonic drag, especially as shown in Fig. 8(a). This corresponds to the phenomena 


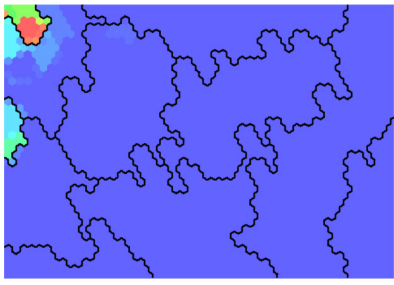

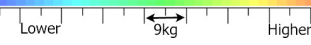

(a) Constraint as wing box volume

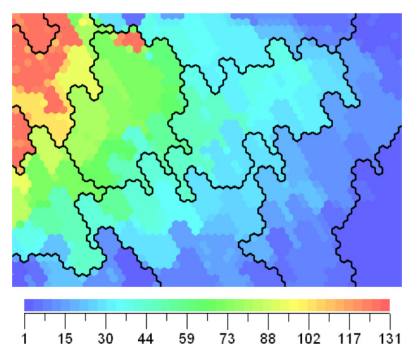

(b) Ranking value in optimizer

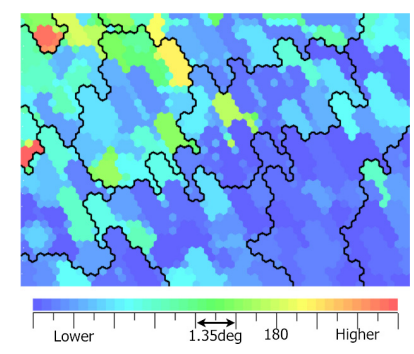

(c) Angle expressing gull-wing

Fig. 5 SOM colored by the characteristic values.

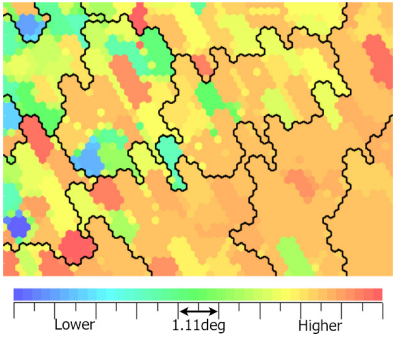

(a) PARSEC $\alpha_{T E}$

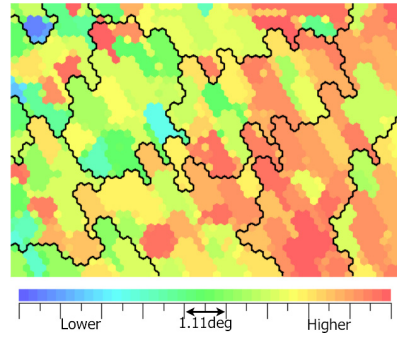

(b) PARSEC $\beta_{T E}$

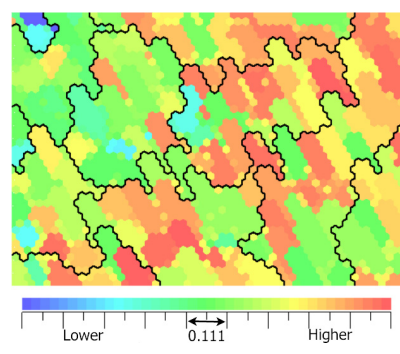

(c) PARSEC $r_{L E_{l o}} / r_{L E_{u p}}$

Fig. 6 SOM colored by characteristic design variables regarding the PARSEC airfoil at $35.0 \%$ spanwise location. The minimum and maximum values of color bar are set using the minimum and maximum values of each design variable in optimizer, respectively.

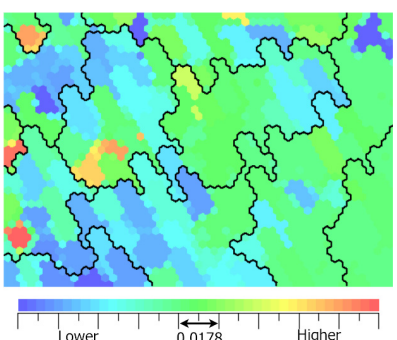

(a) PARSEC $x_{u p}$

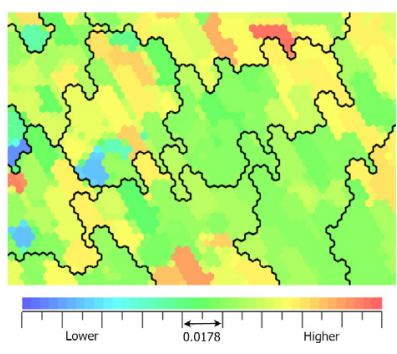

(b) PARSEC $x_{l o}$

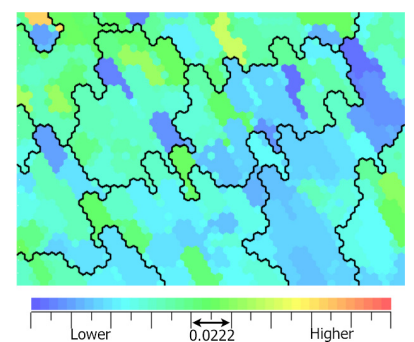

(c) PARSEC $z_{x x_{10}}$

Fig. 7 SOM colored by the characteristic design variables regarding the PARSEC airfoil at $55.5 \%$ spanwise location. The minimum and maximum values of color bar are set using the minimum and maximum values of each design variable in optimizer, respectively.

shown in the CFD visualization. Specifically, the shock wave in the vicinity of the kink is weakened. The angle of attack near the kink with downward twisting is replaced from the initial geometry and the lost lift is made up to replace the angle of attack at the outboard wing with upward twisting so that the wave drag is reduced near the kink. Upward twisting at the outboard wing has no influence on transonic drag, as shown in Fig. 8(d). This corresponds to the CFD prediction. The other design variables were not effective to reduce the objective functions or to increase aerodynamic performance as $C_{D}$ and $L / D$ under transonic cruise flight condition. Data mining techniques using SOM were found to be able to classify the design variables considering their influence on the objectives and aerodynamic performance.

Design knowledge regarding block fuel, which is the most important element of the present optimization problem, will be considered. The following two points are the keys to improve block fuel: 1) $L / D$ increase, 2) $d C_{D} / d \alpha$ increase, at any Mach number. However, there were no single design variable in the present design space capable of satisfying them simultaneously. In fact, this was confirmed by the SOMs. Although PARSEC design variables correspond to aerodynamic performances, there are no direct effects on other objective functions. It would be easier to understand the design space if the design variables have direct 


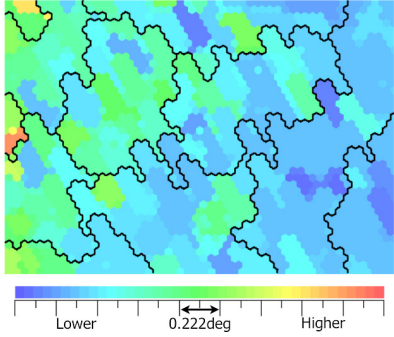

(a) SOM colored by the twist angle at $35.0 \%$ spanwise location

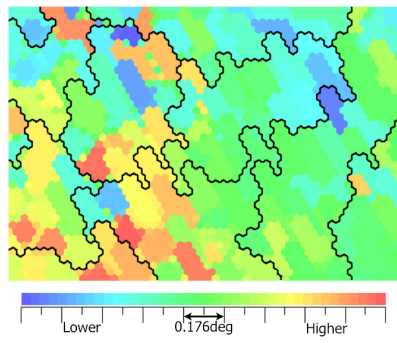

(c) SOM colored by the twist angle at $77.5 \%$ spanwise location

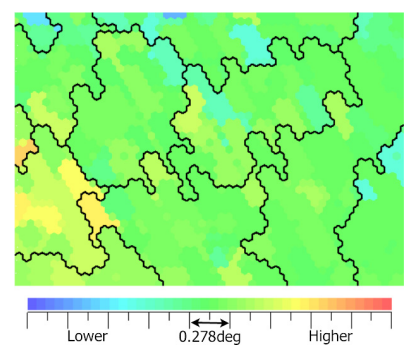

SOM colored by the twist angle at $55.5 \%$ spanwise location

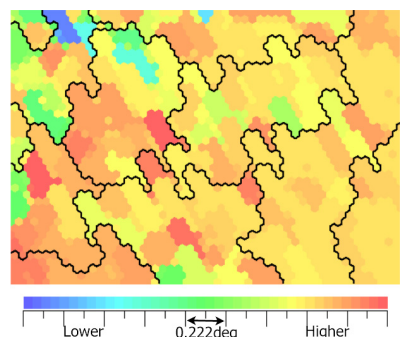

(d) SOM colored by the twist angle at $96.0 \%$ spanwise location

Fig. 8 SOM colored by the characteristic design variables involving wing twist. The minimum and maximum values of color bar are set using the minimum and maximum values of each design variable in optimizer, respectively.

influences on the objective functions.

\subsection{Evaluation of an Improved Geometry}

The design knowledge obtained by data mining shows that a non-gull wing should be designed. Therefore, we modified the optimized wing shape (called as 'optimized' shown in Fig.9 [3]) which achieved the higher improvement in the block fuel to the non-gull wing shape (called as 'optimized_mod') to verify the design knowledge obtained by the previous data mining.

The evaluated results are shown in Figs. 9(a) to 9(c). As already described in Ref. [3], these figures show that optimized_mod improves both block fuel and maximum takeoff weight. Moreover, by comparison of the polar curves at constant $C_{L}$ for cruising condition shown in Fig. 9(d), $C_{D}$ of optimized mod was found to be reduced by 10.6 counts over the initial geometry. Due to the improvement of drag, the block fuel of optimized $\_$mod was reduced by 3.6 percent.

In the present MDE system, surface spline function of the geometry deviation was used for the modification of the wing shape (surface mesh), and then the volume mesh was modified by the unstructured dynamic mesh method. However, this process made the surface mesh distorted around the leading edge and highly limited the design space shown in Fig. 10. This mesh generation process might be the primary reason for the difficulty in finding the non-gull geometry with better block fuel performance. The secondary reason is that only the small number of the generations has been performed. However, this result reveals that data mining technique salvages the information. It is demonstrated that the knowledge discovery by data mining for design space is an important aspect in the practical optimization.

In this study, although MDE is applied for the field of aeronautics, the applicable area is manifold. Nowadays, the application to the internal fan blade of cleaner and the tires for car in industries is heard. When the definition of design problem is performed, MDE can apply to various problems. The application of MDE to conceptual design part can abridge the time cost and design process. Furthermore, the knowledge for innovative design is acquired and the database of design knowledge can be generated. 
Table 2 Effects of design variables to $C_{L}$ under transonic cruising flight condition.

\begin{tabular}{rl|l}
\hline design variable & & $C_{L}$ \\
\hline PARSEC $\alpha_{T E} @ 35.0 \%$ & decrease & increase \\
PARSEC $x_{\text {up }} @ 55.5 \%$ & increase & increase \\
PARSEC $x_{l o} @ 55.5 \%$ & decrease & increase \\
\hline Twist @ $35.0 \%$ & increase & increase \\
Twist @ 55.5\% & increase & increase \\
\hline
\end{tabular}

Table 3 Effects of design variables to $L / D$ under transonic cruising flight condition.

\begin{tabular}{|c|c|c|}
\hline design variabl & & $L / D$ \\
\hline $\begin{array}{c}\text { PARSEC } r_{L E_{I o}} / r_{L E_{u p}} @ 35.0 \% \\
\text { PARSEC } 7\end{array}$ & decrease & decrease \\
\hline
\end{tabular}

Table 4 Effects of design variables to $C_{M p}$ under transonic cruising flight condition.

\begin{tabular}{rl|l}
\hline design variable & & $C_{M p}$ \\
\hline PARSEC $\alpha_{T E} @ 35.0 \%$ & decrease & decrease \\
PARSEC $\beta_{T E} @ 35.0 \%$ & decrease & decrease \\
PARSEC $r_{L E_{l o}} / r_{L E_{u p}} @ 35.0 \%$ & decrease & increase \\
PARSEC $x_{u p} @ 55.5 \%$ & increase & decrease \\
PARSEC $x_{l o} @ 55.5 \%$ & decrease & decrease \\
PARSEC $z_{x x_{l o}} @ 55.5 \%$ & increase & increase \\
\hline
\end{tabular}

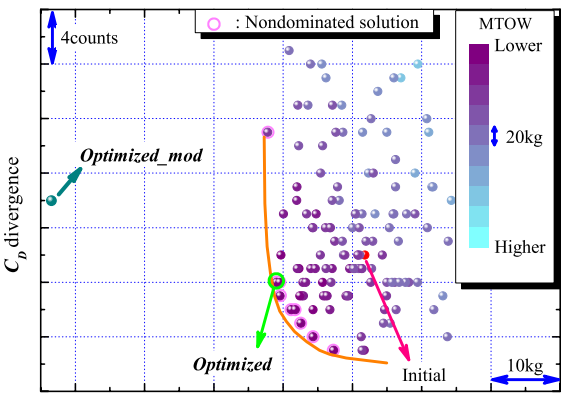

Block Fuel $[\mathrm{kg}]$

(a)

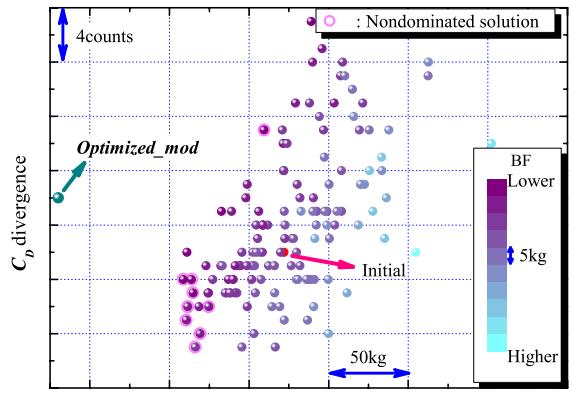

Max Takeoff Weight $[\mathrm{kg}]$

(c)

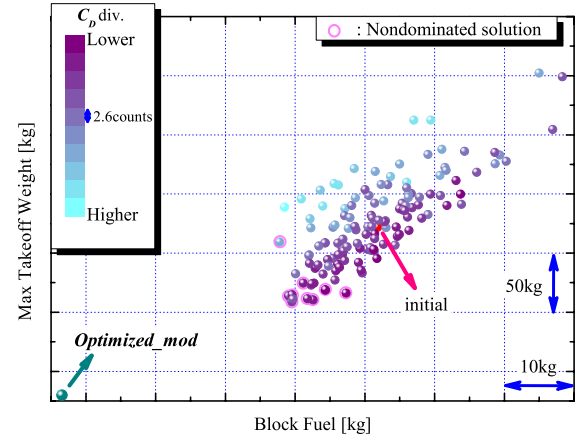

(b)

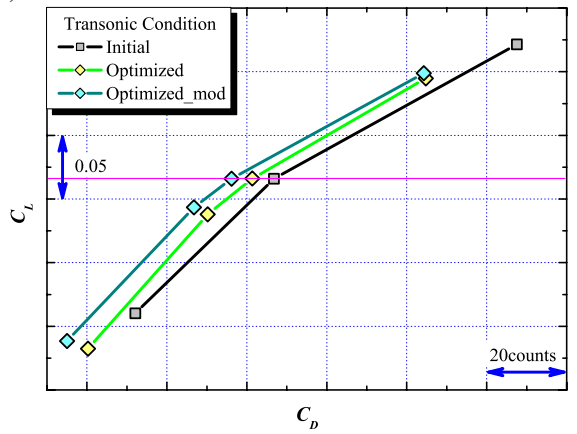

(d)

Fig. 9 Comparison of optimized_mod and the other solutions on two-dimensional plane; (a) between block fuel and $C_{D}$ divergence, (b) between block fuel and maximum takeoff weight, (c) between maximum takeoff weight and $C_{D}$ divergence, (d) comparison of $C_{L^{-}} C_{D}$ curves among initial, optimized, and optimized_mod (from Ref. [3]). 


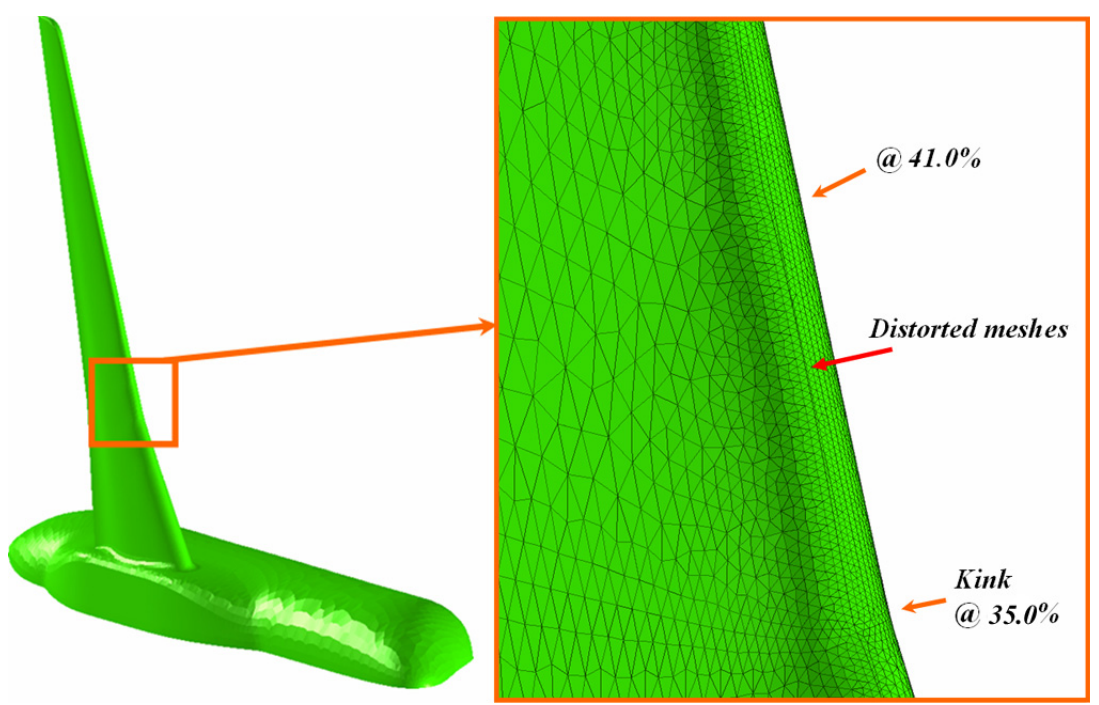

Fig. 10 Example of distorted mesh in the vicinity of leading edge.

\section{Conclusion}

A large-scale and real-world MDE for a transonic regional jet airplane has been performed. The optimization has obtained 130 solutions including nine non-dominated solutions. And then, data mining for the design space has been performed using SOM to provide the design knowledge. As a result, SOM reveals that "which" and "how" design variable influences the objective functions and aerodynamic performances. The higher value of $35 \%$ twist angle increases the maximum takeoff weight. The higher value of 55.5\% twist angle increases the drag divergence. No design variable has direct influence regarding the block fuel. Detailed observations of SOM revealed that there is a sweet spot in the design space where the three objectives become relatively low.

Although the optimization results showed the inverted gull-wings as non-dominated solutions, one of the key features found by data mining was the non-gull wing geometry. When this knowledge was applied to one optimum solution, the resulting design was found to have better performance compared with all solutions included in the non-dominated them and the original geometry designed in the conventional manner. The data mining technique provides knowledge regarding the design space and may salvage lost information during the optimization operation, which will be an important facet of solving practical optimization problems. MDE is also essential for practical aircraft design.

\section{Acknowledgment}

The Euler and N-S computations were performed using NEC SX-5 in the Institute of Fluid Science, Tohoku University, and NEC SX-7 in Super Computing System Information Synergy Center, Tohoku University.

\section{References}

( 1 ) Holden, C. M. E. and Keane, A. J., "Visualization Methodologies in Aircraft Design," AIAA Paper 2004-4449, 2004.

( 2 ) Obayashi, S., Jeong, S., and Chiba, K., "Multi-Objective Design Exploration for Aerodynamic Configurations," AIAA Paper 2005-4666, 2005.

( 3 ) Obayashi, S., Jeong, S., Chiba, K., and Morino, H., "Multi-Objective Design Exploration and Its Application to Regional-Jet Wing Design," Transactions of the Japan Society for Aeronautical and Space Sciences, Vol. 50, No. 167, 2007, pp. 1-8.

( 4 ) Kohonen, T., Self-Organizing Maps, Springer, Berlin, Heidelberg, 1995. 
( 5 ) Vesanto, J. and Alhoniemi, E., "Clustering of the Self-Organizing Map," IEEE Transactions on Neural Networks, Vol. 11, No. 3, 2000, pp. 586-600.

( 6 ) Chiba, K., Obayashi, S., Nakahashi, K., and Morino, H., "High-Fidelity Multidisciplinary Design Optimization of Aerostructural Wing Shape for Regional Jet," AIAA Paper 2005-5080, 2005.

( 7 ) Oyama, A., Obayashi, S., Nakahashi, K., and Hirose, N., "Aerodynamic Wing Optimization via Evolutionary Algorithms Based on Structured Coding," Computational Fluid Dynamics Journal, Vol. 8, No. 4, 2000, pp. 570-577.

( 8 ) Yamazaki, W., Matsushima, K., and Nakahashi, K., "Aerodynamic Optimization of NEXST-1 SST Model at Near-Sonic Regime," AIAA Paper 2004-0034, 2004.

( 9 ) Sasaki, D. and Obayashi, S., "Efficient Search for Trade-Offs by Adaptive Range MultiObjective Genetic Algorithms," Journal of Aerospace Computing, Information, and Communication, Vol. 2, No. 1, 2005, pp. 44-64.

(10) Krishnakumar, K., "Micro-Genetic Algorithms for Stationar and Non-Stationary Function Optimization," SPIE 1196, Proceedings of Intelligent Control and Adaptive Systems, 1989, pp. 289-296.

(11) Fonseca, C. M. and Fleming, P. J., "Genetic Algorithms for Multiobjective Optimization: Formulation, Discussion and Generalization," Proceedings of the Fifth International Conference on Genetic Algorithms, 1993, pp. 416-423.

(12) Obayashi, S., Takahashi, S., and Takeguchi, Y., "Niching and Elitist Models for MOGAs, Parallel Problem Solving from Nature," The 5th International Conference onParallel Problem Solving from Nature, LNCS 1498, Springer, Berlin, Heidelberg, New York, 1998, pp. 260-269.

(13) Baker, J. E., "Reducing Bias and Inefficiency in the Selection Algorithm," Proceedings of the Second International Conference on Genetic Algorithms, 1987, pp. 14-21.

(14) Eshelman, L. J. and Schaffer, J. D., "Real-Coded Genetic Algorithms and Interval Schemata," Foundations of Genetic Algorithms 2, Morgan Kaufmann, San Mateo, CA, 1993, pp. 187-202.

(15) Deb, K., Multi-Objective Optimization Using Evolutionary Algorithms, John Wiley \& Sons, Ltd., Chichester, 2001.

(16) “MSC. website,” URL: http://www.mscsoftware.com/ [cited 14 September 2004].

(17) Ito, Y. and Nakahashi, K., "Direct Surface Triangulation Using Stereolithography Data," AIAA Journal, Vol. 40, No. 3, 2002, pp. 490-496.

(18) Ito, Y. and Nakahashi, K., "Improvements in the Reliability and Quality of Unstructured Hybrid Mesh Generation," International Journal for Numerical Methods in Fluids, Vol. 45, Issue 1, 2004, pp. 79-108.

(19) “Eudaptics website,” URL: http://www.eudaptics.com [cited 16 June 2004].

(20) Deboeck, G. and Kohonen, T., Visual Explorations in Finance with Self-Organizing Maps, London, Springer Finance, 1998.

(21) Pampalk, E., Rauber, A., and Merkl, D., "Content-Based Organization and Visualization of Music Archives," Proceedings of the $10^{\text {th }}$ International conference on Multimedie, ACM Press, NY, 2002, pp. 570-579.

(22) Obayashi, S. and Sasaki, D., "Visualization and Data Mining of Pareto Solutions Using Self-Organizing Map," The 2nd International Conference on Evolutionary MultiCriterion Optimization, LNCS 2632, Springer-Verlag Heidelberg, Faro, Portugal, 2003, pp. 796-809.

(23) Chiba, K., Jeong, S., Obayashi, S., and Morino, H., "Data Mining for Multidisciplinary Design Space of Regional-Jet Wing," Proceedings on IEEE Congress on Evolutionary Computation 2005, 2005, pp. 2333-2340. 